\title{
Correlation between S'-Wave Dispersion of Mitral Annular Tissue Velocity and Severity of Coronary Artery Disease Assessed by SYNTAX Score
}

\author{
MOHAMMED NAGIB, M.D.; KAMAL MARGHANY, M.D.; MOHAMMED ABD EL-HADY, M.D. and \\ AHMAD KHAIRY, M.Sc.
}

The Department of Cardiology, Faculty of Medicine, Al-Azhar University, Cairo

\begin{abstract}
Background: Coronary Artery Disease (CAD) is a paramount cause of death. Coronary angiography is considered the gold standard for the invasive assessment of obstructive CAD. The SYNTAX score is frequently used to comprehensively assess the complexity and burden of CAD. The technique of Tissue Doppler Imaging (TDI) emerged as a unique, easily performed, and reproducible modality for assessing systolic and diastolic LV performance. We aimed to examine the value of the $\mathrm{S}^{\prime}$-wave dispersion of TDI derived mitral annular velocities for the prediction of severity of coronary artery stenosis.
\end{abstract}

Methods: We included 60 patients with symptoms suggesting CAD in the study. We excluded patients with previous myocardial infarction, atrial fibrillation, significant valvular disease, and congestive heart failure. All patients had undergone full history taken and clinical examination; complete 12-leads electrocardiography, echocardiographic with assessment of LV systolic and diastolic dimensions, fraction of shortening, ejection fraction, and Doppler derived mitral valve velocities; TDI with measuring of $\mathrm{S}^{\prime}$-wave, $\mathrm{S}^{\prime}$-wave dispersion, $\mathrm{S}^{\prime}$ mean, E'-wave, $\mathrm{A}^{\prime}$-wave and $\mathrm{E}^{\prime} / \mathrm{A}^{\prime}$ ratio of the septal, lateral, anterior and inferior walls; and coronary angiography.

Results: Mean S' was decreased in patients with severe $\mathrm{CAD}$ and $\mathrm{S}^{\prime}$-wave dispersion was high in patients with single or two vessel disease and low in patients with multi vessel disease.

Conclusion: $\mathrm{S}^{\prime}$-wave dispersion in combination with $\mathrm{S}^{\prime}$ mean can predict severity of CAD and number of affected vessel.

Key Words: Tissue Doppler imaging - Mitral annular velocities - $S^{\prime}$-wave dispersion - Coronary artery disease - Coronary angiography - Syntax score.

\section{Introduction}

CORONARY Artery Disease (CAD) is the leading cause of death worldwide. The World Health Or-

Correspondence to: Dr. Mohammed Nagib, The Department of Cardiology, Faculty of Medicine, Al-Azhar University, Cairo ganization estimates that approximately 17 million people die from CAD every year [1]

An estimated 84 million American adults are afflicted with cardiovascular disease, accounting for 1 in 3 individuals. This number is expected to rise given the increasing rates of obesity and diabetes leading to greater economic strain on our overall health care system [2].

Coronary angiography is considered the gold standard for the invasive assessment of obstructive CAD. While generally considered a safe procedure, coronary angiography is associated with complications. Major complications including death, Myocardial Infarction (MI), and/or major embolization have been estimated to occur in less than $2 \%$ of cases [3].

The SYNTAX score is frequently used to comprehensively assess the complexity and burden of CAD [4]

It was initially developed to assess outcomes in patients with left main or three-vessel disease. The SYNTAX score is an anatomically based risk calculation that was developed as an aid in determining the optimal technique of revascularization. It has been proven to predict prognosis and response to revascularization strategies [5]

Global and regional Left Ventricular (LV) systolic function is an important marker of CAD in echocardiographic studies, which is usually assessed using two-dimensional echocardiography [6].

The technique of Tissue Doppler Imaging (TDI) emerged as a unique, easily performed, and repro- 
ducible modality for assessing systolic and diastolic LV performance [7].

By using TDI, we can visualize and measure the low systolic and diastolic velocities generated by myocardial movements [8]

Using the pulsed wave Doppler, the myocardium generates the following measures: S'-wave (systolic wave), E'-wave (early diastolic wave), and $\mathrm{A}^{\prime}$-wave (late diastolic wave).

Other measures like E'/A' (the ratio of early and late diastolic tissue velocity as measured by $\mathrm{TDI}$ ), and $\mathrm{E} / \mathrm{E}^{\prime}$ (the ratio of early diastolic transmitral flow velocity and early diastolic tissue velocity), may also be taken for evaluating $\mathrm{LV}$ diastolic function [9].

However, the cutoff values for maximum systolic velocity and diastolic velocities which may detect CAD are unclear [8].

Also associated diseases, like diabetes mellitus [10] or hypertension [11] have an adverse effect on global systolic and diastolic functions of the LV which may make the use of TDI derived velocities for diagnosis of CAD more difficult. So, we aimed at studying the value of the $\mathrm{S}^{\prime}$-wave dispersion of TDI derived mitral annular velocities for the prediction of severity of coronary artery stenosis.

\section{Patients and Methods}

This study had been carried out at our Outpatient Clinics at Bab El-She'riya and Al-Hussein University Hospitals, Al-Azhar University, Cairo, Egypt. We included 60 patients (40 males and 20 females) between February 2017 and August 2017 with symptoms suggesting CAD in whom coronary angiography was indicated according to the 2012 ACCF/AHA/ACP/AATS/PCNA/SCAI/STS Guideline for the diagnosis and management of patients with stable ischemic heart disease [12] patients were excluded from the study if they had one or more of the following:

- Diabetic and hypertensive patients.

- Patients with previous Coronary Artery Bypass Surgery (CABG) as the SYNTAX score cannot be applied in this patient population.

- Previous Myocardial Infarction (MI).

- Patients with significant valvular heart disease (more than mild stenosis or regurgitation).

- Left ventricular ejection fraction $\leq 50 \%$.
- Significant regional wall motion abnormalities.

- Congestive heart failure.

- Atrial fibrillation.

- Congenital heart disease.

- Technically poor acoustic window for transthoracic echocardiography.

After giving an informed written consent, we did the following to all patients:

- Full history taking and thorough clinical examination.

- Complete 12-leads electrocardiography.

- Echocardiography: Echocardiographic and Doppler studies were performed for all patients using Philips IE 33 X Matrix phased array system equipped with TDI and STE technology, using a multi frequency $(1-5 \mathrm{MHz}) \mathrm{S} 5-1$ matrix array probe was used. The studies were performed by two expert operators unaware of the patients' clinical data and of each other measures. The following measures were taken: Two-dimensional guided M-mode measurements of Left Ventricular End-Diastolic Dimension (LVEDD), Left Ventricular End-Systolic Dimension (LVESD), Ejection Fraction (EF) and Fraction of Shortening (FS) [13]

Doppler derived MV flow velocity waves; Ewave, A- wave, and E/A ratio [13].

Tissue-Doppler Imaging (TDI): Myocardial velocities of MV annulus were measured to all subjects at apical 4-chamber and apical 2-chamber views. We measured the velocities at the septal, lateral, anterior, and inferior walls of mitral valve annulus. Three velocities were taken for every wall, S'-wave for systole, and two waves for diastole; E'-wave at early filling phase, and $\mathrm{A}^{\prime}$-wave for atrial contraction phase [14] and mean $S^{\prime}$ is calculated. S'-wave dispersion was calculated for every patient according to the following formula: $\mathrm{S}^{\prime}$-wave dispersion=(Maximum S'-Minimum $\left.\mathrm{S}^{\prime}\right) /$ Maximum S'\%.

- Coronary angiography:Coronary angiography was performed by the percutaneous femoral or radial approach. Two idependant clinicians blinded to the echocardiographic results reviewed the coronary angiography of each patient to calculate the SYNTAX score according to SYNTAX score algorithm and patients were be categorized as having a low SYNTAX score $(\leq 22)$, intermediate score (23-32), or high score $(\geq 33)$. 
Student's $t$-test (two-tailed), Chi-square $\left(\chi^{2}\right)$, one-way ANOVA and Fisher's exact (if required) tests were used for comparison between variables. $p$-value $<0.01$ is considered highly statistically significant result. Pearson correlation coefficient $(r)$ was used as a measure of the strength of correlation between two variables. Spearman's rank correlation was performed if skewed variables.

\section{Results}

We identified 60 patients who met the inclusion and exclusion criteria.

Male patients represented the higher.

Proportion (66.7\% male and $33.3 \%$ female). The mean \pm SD age of the patients in the studied sample was $56.87 \pm 10.92$ years. While the mean \pm SD BMI was $30.65 \pm 7.36 \mathrm{~m}^{2}$ and the mean \pm SD BSA was $2.0 \pm 0.12 \mathrm{~kg} / \mathrm{m}^{2}$ (Table 1).

The patients were classified into 3 groups according to SS as flow.

- Group I: Included 35 patients with low SYNTAX score $(\leq 22)$.

- Group II: Included 15 patients with intermediate score (23-32).

- Group III: Included 10 patients with high score $(\geq 33)$.

Table (1): Demographic data distribution of the study group.

\begin{tabular}{lll}
\hline Demographic data & No. & $\%$ \\
\hline Male & 40 & 66.7 \\
Female & 20 & 33.3 \\
& Range & Mean \pm SD \\
Age (years) & $33-80$ & $56.87 \pm 10.92$ \\
Weight $(\mathrm{kg})$ & $70-110$ & $86.98 \pm 12.79$ \\
Height $(\mathrm{cm})$ & $150-191$ & $169.52 \pm 10.39$ \\
BMI $\left[\mathrm{wt} /(\mathrm{ht})^{2}\right]$ & $22.86-46.9$ & $30.65 \pm 7.36$ \\
BSA & $1.83-2.3$ & $2.0 \pm 0.12$ \\
\hline
\end{tabular}

Table (2) summarizes echocardiographic data in each of the studied group. There was a statistically significant difference as regard EF, Doppler mitral inflow and TDI parameters.

As shown in the Fig. (1) there was negative correlation between mean $\mathrm{S}^{\prime}$ and SS.

So we can predict severity of CAD by measuring mean $S^{\prime}$ but there was no correlation between $\mathrm{S}^{\prime}$ wave dispersion and SS as shown in the Fig. (2), $(p=0.123)$.
Table (2): Echocardiographic data of the study group.

\begin{tabular}{llllc}
\hline & Group I & Group II & Group III & $p$-value \\
\hline LVEDD & $5.02 \pm .32$ & $5.04 \pm .32$ & $5.11 \pm .17$ & .741 \\
LVESD & $3.23 \pm .29$ & $3.35 \pm .31$ & $3.3 \pm .26$ & .319 \\
IVS & $.95 \pm .12$ & $.97 \pm .69$ & $.93 \pm .10$ & .700 \\
EF & $60.86 \pm 2.27$ & $55.80 \pm 2.2$ & $55.00 \pm 1.87$ & .0001 \\
AoR & $3.02 \pm .35$ & $2.90 \pm .31$ & $2.93 \pm .37$ & .525 \\
LA & $3.67 \pm .62$ & $3.96 \pm .53$ & $3.90 \pm .65$ & .267 \\
E/A & $.88 \pm .35$ & $1.13 \pm .62$ & $1.2 \pm .60$ & .037 \\
e & $6.98 \pm 1.60$ & $5.97 \pm 1.52$ & $5.78 \pm 1.06$ & .027 \\
a & $8.26 \pm 2.13$ & $7.88 \pm 2.43$ & $7.71 \pm 2.66$ & .742 \\
E/e & $9.80 \pm 4.49$ & $13.97 \pm 6.64$ & $14.18 \pm 5.22$ & .011 \\
S' septal & $9.61 \pm 1.59$ & $6.20 \pm 1.64$ & $6.70 \pm 1.64$ & .0001 \\
S' lateral & $10.73 \pm 2.28$ & $6.93 \pm 1.04$ & $6.57 \pm 1.40$ & .0001 \\
S' anterior & $10.46 \pm 1.73$ & $6.19 \pm .96$ & $6.09 \pm 1.23$ & .0001 \\
S' inferior & $11.09 \pm 1.84$ & $8.24 \pm 2.28$ & $8.88 \pm 2.79$ & .0001 \\
Mean S' & $10.51 \pm .76$ & $7.06 \pm .81$ & $6.80 \pm 1.05$ & .0001 \\
S' dispersion & $33.19 \pm 11.57$ & $33.85 \pm 14.27$ & $38.96 \pm 15.60$ & .465 \\
\hline
\end{tabular}

LVEDD : Left Ventricular End Diastolic Dimensions.

LVESD : Left Ventricular End Systolic Dimensions.

EF : Ejection Fraction.

IVS : Interventricular Septum.

AoR : Aortic Root.

LA : : Left Atrium.

S : Systolic.

E : Refers to early filling phase.

A : Refers to atrial contraction phase.

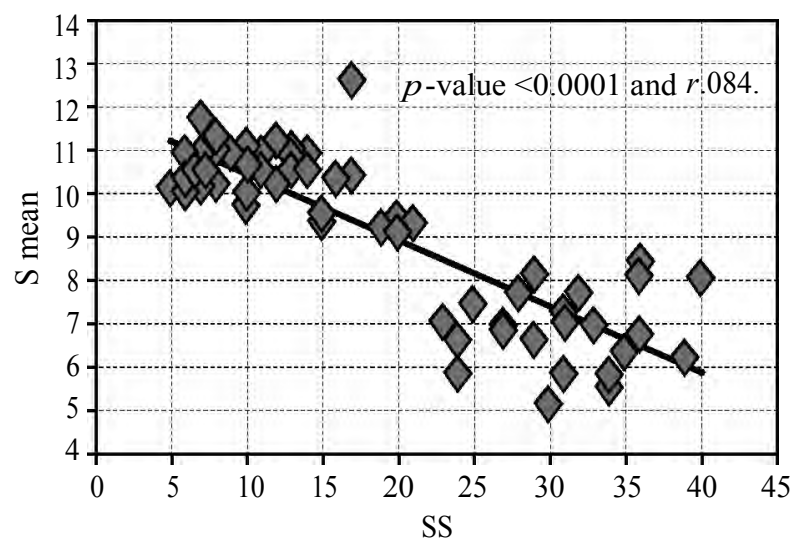

Fig. (1): Linear correlation between SS and S' mean in the study population.

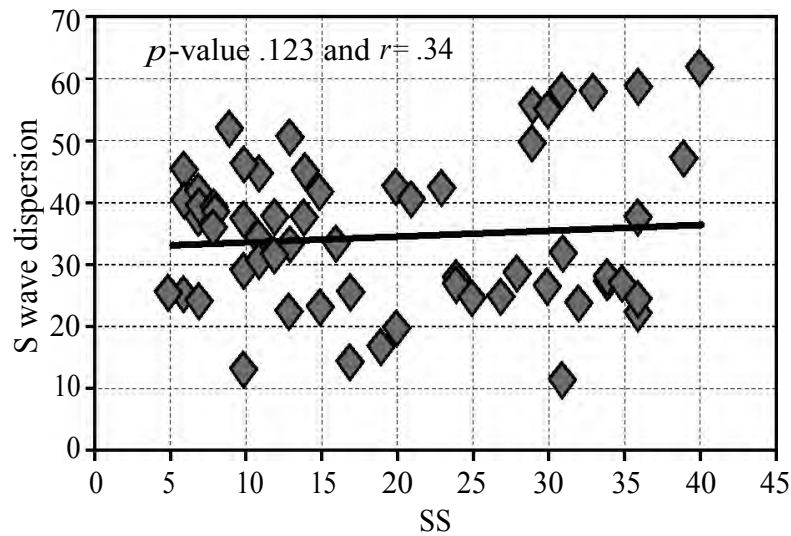

Fig. (2): Linear correlation between $\mathrm{S}^{\prime}$ wave dispersion and SS 
But measuring $\mathrm{S}^{\prime}$ wave dispersion we determine if the patient had single vessel disease, two vessel disease or multi vessel disease, with a sensitivity and a specificity of $80 \%$ and $71 \%$ respectively, and cut off value of 28.45 . Patients with $\mathrm{S}^{\prime}$ wave dispersion less than 28.45 were found to be with MVD, and more than 28.45 found to be with single or two vessel disease as shown in the Fig. (3).

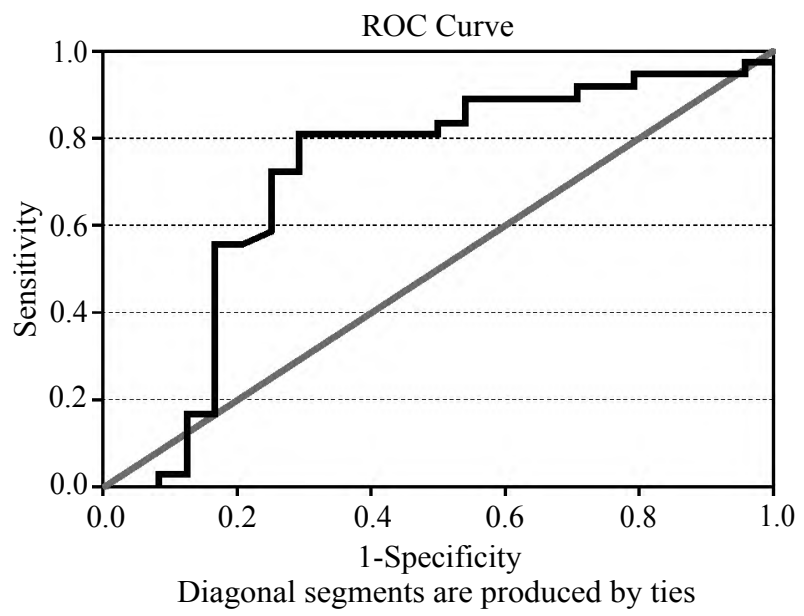

Fig. (3): ROC curve S' wave dispersion.

\section{Discussion}

The prevalence of Coronary Artery Disease (CAD) continues to rise worldwide associated with increasing patient morbidity and mortality.

In the early phase of Ischemic Heart Disease (IHD), diastolic dysfunction can be detected noninvasively by TTE prior to the development of overt systolic dysfunction.

Visual detection of segmental all motion abnormality is the basic method in diagnosis of CAD by echocardiography [15].

However, detection of wall motion abnormalities, based only on visual assessment has many limitations being a qualitative method and totally subjective method that depends to a great extent on operator experience and the quality of the images [16].

Tissue Doppler imaging has been introduced for quantification of regional and global, systolic and also diastolic LV functions. During ischemic events, the longitudinal endocardial fibers are the first being affected; then velocity changes can be detected from the apical approach. In addition to systolic velocity $\mathrm{S}^{\prime}$, diastolic velocity $\mathrm{E}^{\prime}$ and $\mathrm{E}^{\prime} / \mathrm{A}^{\prime}$ ratio were found to be correlating with wall motion abnormalities [17]. Derumeaux and his colleagues [18] have found a strong correlation between myo- cardial systolic velocity and regional myocardial blood flow in animal models of myocardial ischemia.

Similar results were found in humans as Katz and his colleagues [19] have found that decreased maximum systolic velocity was a highly sensitive and specific figure for detection of abnormal segmental myocardial motion and was able to diminish the variability in the visual interpretation of wall motion abnormalities assessed during dobutamine stress echocardiography.

Our study demonstrated that patients with high CAD burden based on SYNTAX score have lower EF as compared with patients with low/intermediate syntax score. We found that there was strong negative correlation between EF and SS with $p$-value $<0.001$.

Also, our study demonstrated that patients with high CAD burden based on SYNTAX Score have lower mean S' as compared with patients with low/ intermediate syntax score. We found that there was strong negative correlation between mean $\mathrm{S}^{\prime}$ and SS with $p$-value $<0.0001$.

Our study demonstrated that higher E/A ratio was predictive of higher CAD burden based on the SYNTAX score with $p$-value $<0.037$.

Bruch et al., examined the E/A ratio in a patient population with single vessel CAD in the setting of preserved LVEF. They demonstrated that the E/A ratio was significantly lower in patients with $\mathrm{CAD}$, mainly due to lower E-wave velocities [20].

In a study by Sun et al., [21], they found that $\mathrm{E} / \mathrm{E}^{\prime}$ at rest was a good indicator of significant CAD detection. They found that a cutoff point of $\mathrm{E} / \mathrm{E}^{\prime}>8.34$ was able to detect $\mathrm{CAD}$ with an accuracy of $85.8 \%$, sensitivity $77.4 \%$ and specificity $100 \%$.

In our study we also found that $\mathrm{E} / \mathrm{E}^{\prime}$ at rest was a good indicator of significant $\mathrm{CAD}$ detection with $p$-value $<0.011$.

Most of the studies on TDI were done on relatively small number of patients and showed significant heterogeneity of their measured velocities in normal or abnormal segments. This heterogeneity was observed in the meta-analyses of maximum early and late diastolic velocity (pre-stress); and maximum systolic velocity and late diastolic velocity (post-stress). This heterogeneity could be the result of subtle differences in patient populations, segments in which TDI velocities were measured, models of the echocardiography machines used in the assessments, or type of tissue Doppler 
used (spectral Doppler, which measures the instantaneous velocity, versus color Doppler which measures the modal velocity) [22]

In our study we found that $S^{\prime}$ wave dispersion is lower in patient with MVD as compared to patient with single and two vessel disease. Patients with $\mathrm{S}^{\prime}$ wave dispersion $<28.45$ found to be with MVD and patients with $\mathrm{S}^{\prime}$ wave dispersion >_28.45 found to be with single or two vessel disease.

In a study by Aldaydamony it was founded that S' wave dispersion may predict significant CAD but in our study we founded that it can't be applied to patients with MVD as S'wave dispersion is decreased in those patients [23]

\section{Conclusion:}

The mean $\mathrm{S}^{\prime}$ may be a good predictor for angiographically severe CAD assessed by SS and S' wave dispersion may be a good predictor of number of vessel affected as a cutoff point of $<28.45$ with MVD. Further studies on larger number of patients and on other patients' categories are recommended.

\section{Study limitations:}

In addition to the relatively small number of patients and being a single center study, the major limitation of our study is that it did not include many patients' categories like those with previous MI, diabetic patients, hypertensive patients, those with $\mathrm{AF}$ and those with $\mathrm{CHF}$. We did not include these categories because of the effect of MI on LV regional systolic and diastolic functions beyond the presence of flow limiting coronary obstruction and the variability of TDI velocities with AF.

\section{Disclosures:}

The authors declare that there is no conflict of interest.

\section{References}

1- World Health Organization: The Atlas of Heart Disease and Stroke: Available at http://www.who.int/cardiovascular diseases/resources/atlas/en/. (accessed December 10, 2016), 2014.

2- GO A.S., MOZAFFARIAN D., ROGER V.L., et al.: Heart disease and stroke statistics-2014 update: A Report From the American Heart Association. Circulation, 129: e28e292, 2014.

3- SCANLON P.J., FAXON D.P. and AUDET A.M : ACC/AHA guidelines for coronary angiography: Executive summary andrecommendations: A report of the American College of Cardiology/American Heart Association task force on practice guidelines (committee on coronary angiography) developed in collaboration with the Society for Cardiac Angiography and Interventions. Circulation; 2345-57, 1999.
4- SIANOS G., MOREL M.A. and KAPPETEIN A.P.: The SYNTAX Score: An angiographic tool grading the complexity of coronary artery disease. Euro. Intervention, 1 : 219-27, 2005

5- HE J.Q., GAO Y.C., YU X.P., et al.: Syntax score predicts clinical outcome in patients with three-vessel coronary artery disease undergoing percutaneous coronary intervention. Chin. Med. J. (Engl.), 124: 704-9, 2011.

6- OH J.K., APPLETON C.P., HATLE L.K., et al.: The noninvasive assessment of left ventricular diastolic function with two-dimensional and Doppler echocardiography. J. Am. Soc. Echocardiogr., 10: 246-70, 1997.

7- LEUNG D.Y. and NG A.C.: Emerging clinical role of strain imaging in echocardiography. Heart Lung Circ., 19: 161-74, 2010.

8- AGARWAL R., GOSAIN P., KIRKPATRICK J.N., et al.: Tissue Doppler imaging for diagnosis of coronary artery disease: A systematic review and meta-analysis. Cardiovascular Ultrasound, 10: 47-55, 2012.

9- YANG L., WU W., WANG J.F., et al.: Quantification of global left ventricular systolic dysfunction in patients with coronary artery disease by pulsed Doppler tissue imaging: The value of mitral annulus time intervals. Echocardiography, 24: 360-5, 2007.

10- MURARKA S. and MOVAHED M.R.: Diabetic cardiomyopathy. J. Card. Fail., 16: 971-9, 2010.

11- De CARVALHO FRIMM C., SOUFEN H.N., KOIKE M.K., et al.: The long-term outcome of patients with hypertensive cardiomyopathy. J. Hum. Hypertens., 19: 393-400, 2005.

12- FIHN S.D., GARDIN J.M., ABRAMS J., BERRA K., et al.: ACCF/AHA/ACP/AATS/PCNA/ SCAI/STS Guideline for the diagnosis and management of patients with stable ischemic heart disease: A report of the American College of Cardiology Foundation/American Heart Association Task Force on Practice Guidelines, and the American College of Physicians, American Association for Thoracic Surgery, Preventive Cardiovascular Nurses Association, Society for Cardiovascular Angiography and Interventions, and Society of Thoracic Surgeons. J. Am. Coll. Cardiol., 60: e44-e 164, 2012.

13- ARMSTRONG W.F.: Echocardiography. In: Braunwald E., Libby P., Bonow R.O., Mann D.L., Zipes D.P., editors. Heart Disease: A textbook of Cardiovascular Medicine (8th edt.). W.B. Saunders: Philadelphia; p. 187, 2008.

14- HO C.Y. and SOLOMON S.D.: A Clinician's Guide to Tissue Doppler Imaging. Circulation, 113; e396, 2006.

15- SCHILLER N.B., SHAH P.M., CRAWFORD M., DeMARIA A., DEVEREUX R., et al.: Recommendations for quantitation of the left ventricle by two-dimensional echocardiography. J. Am. Soc. Echocardiogr., 2: 358-67, 1989.

16- PICANO E., LATTANZI F., ORLANDINI A., MARINI C., et al.: Stress echocardiography and the human factor: The importance of being expert. J. Am. Coll. Cardiol., 17: 666-9, 1991.

17- MUNDIGLER G. and ZEHETGRUBER M.: Tissue Doppler imaging: Myocardial velocities and strain-are there clinical applications? J. Clin. Basic Cardiol., 5: 125-32, 2002. 
18- DERUMEAUX G., OVIZE M., LOUFOUA J., ANDREFOUET X., et al.: Doppler tissue imaging quantitates regional wall motion during myocardial ischemia and reperfusion. Circulation, 97: 1970-7, 1998.

19- KATZ W.E., GULATI V.K., MAHLER C.M. and GORCSAN J. $3^{\text {rd: }}$ Quantitative evaluation of the segmental left ventricular response to dobutamine stress by tissue Doppler echocardiography. Am. J. Cardiol., 79: 1036-42, 1997.

20- BRUCH C., SCHMERMUND A., BARTEL T., et al.: Tissue Doppler Imaging (TDI) for on-line detection of regional early diastolic ventricular asynchrony in patients with coronary artery disease. Int. J. Card. Imaging, 15 379-90, 1999.
21- SUN L., MA C., LIU S., ZOU L. and JIA D.: Mitral annular tissue velocity in the diagnosis of coronary artery disease. Eur. Rev. Med. Pharmaco. Sci., 18: 3754-60, 2014.

22- AGARWAL R., GOSAIN P., KIRKPATRICK J.N., ALYOUSEF T., et al.: Tissue Doppler imaging for diagnosis of coronary artery disease: A systematic review and metaanalysis. Cardiovascular Ultrasound, 10: 47-55, 2012.

23- AL-DAYDAMONY M. and MUSTAFA T.: Value of S wave dispersion of mitral annular tissue velocity in predicting the presence of significant coronary artery stenosis. I.J.S.R., 5: 1409-13, 2016.

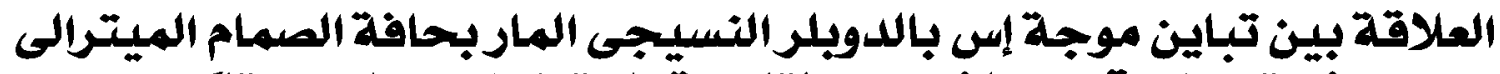

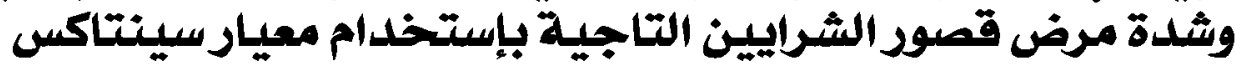

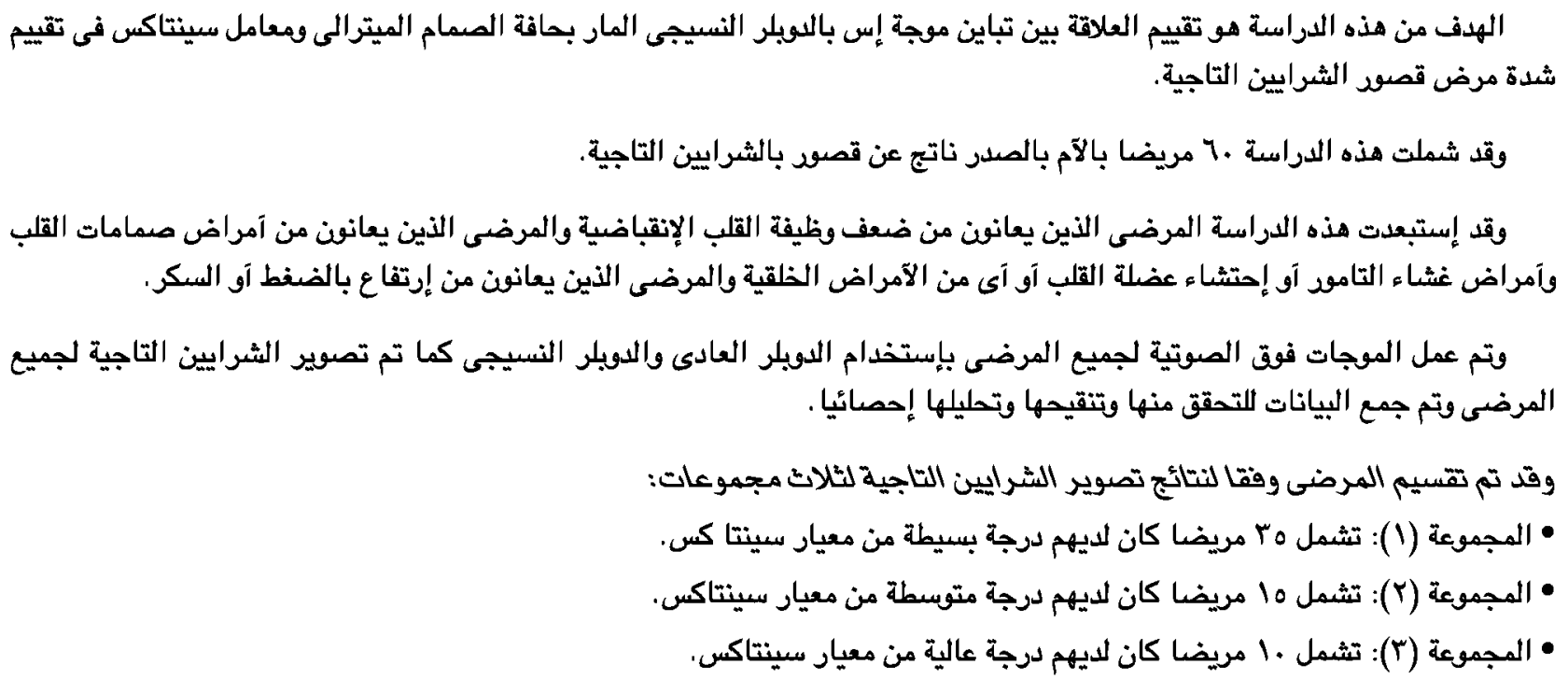

